\title{
Inspection of Pseudo Kissing-bond Defects in Composite Laminate with Infrared Sensing
}

\author{
Liping Zhao ${ }^{1^{\star}}$, Wenjiang Guo ${ }^{1,2}$ \\ *Corresponding author: zhao liping@nmc.a-star.edu.sg \\ ${ }^{1}$ National Metrology Centre, 1 Science Park Drive, Singapore 118221 \\ ${ }^{2}$ School of Mechanical and Aerospace Engineering, Nanyang Technology University, \\ 50 Nanyang Avenue, Singapore 639798
}

\begin{abstract}
Nowadays, composite materials are increasingly being used in aerospace and automobile industries because of its better weight over cost ratio than the conventional metals, easier manufacturing process to fabricate complex geometry, and less maintenance needed during service. But not many traditional non-destructive testing techniques are workable and effective for special composite defects, such as kissing-bond existing in composite laminates which is difficult to be detected through traditional non-destructive testing methods like ultrasonic scanning. This paper proposed and experimentally investigated transient infrared sensing method to detect pseudo-kissing bonds in glass fibre and carbon-fiber composite specimens. The effects of various parameters in system configurations, as in infrared signal path arrangement by reflection or transmission, the rate of heating, and the presence of adhesives in simulating kissing bonds were investigated and the results are presented in the paper. This study demonstrated the potential of the transient infrared sensing for kissing bond detection of composite.
\end{abstract}

Keywords:

Thermal NDT, kissing bonds, carbon-fiber laminates, glass-fiber laminate, transient infrared imaging, thermal signature

\section{INTRODUCTION}

The composite materials have been widely used in the new generation of airplanes such as Boeing $787^{[1]}$ and Airbus 350 owing to the strong urge for higher efficiency and performance in the aviation industries. Advanced composite laminates and composite sandwich will be used extensively in both of these models of commercial airplanes, shifting the main element of the aircraft from aluminium alloys to composite materials. The shift in material selection will increase the strength-to-weight ratio, corrosion resistance, stiffness and fuel efficiency, just to name a few [2]. Imperfect manufacturing conditions can lead to flaws and defects in a fabricated composite. Commonly encountered flaws in composites are delaminations, cracks, disbonds, voids, porosity, inclusion, matrix cracking, fiber waviness, fiber and ply misalignments and kissing bonds[3]. Kissing bonds are recognized as the defects in laminar composites whereby the surfaces of the laminates are in close physical contact with each other, but there is little to no bonding strength between the surfaces [4]. Although there are a few literature published [5-9] on the subject, there is no complete understanding on the formation of kissing bonds.

With the dramatic increase of composite usage in the manufacturing of airplanes, non-destructive inspection and evaluation requirements are becoming increasingly important and hot topic in both the 
manufacturing of the aircraft structures and the subsequent in-services maintenance and repair. As the surfaces are in physical contact with each other and there is no separation, conventional NDE techniques such as ultrasound testing are unable to accurately and reliably detect the presence of the kissing bonds [4,5]. Literatures have tried to detect kissing bonds through several existing methods such as Lamb waves, vibration measurements, modification to ultrasonic techniques and sonic thermography. However, the existing techniques do not allow large scale detection of kissing bonds in a more practical and economical manner. Thus, new methods need to be developed to detect these defects so that composites can be used safely in commercial and industrial applications. Kissing bonds are simulated using various methods. Examples of methods used to simulate kissing bonds are drilling flat-bottom holes in the structure [4], introducing a dry layer of silicone or electrically disbonding the epoxy [5].

Thermography involves the detection of flaws by monitoring the transient heat flow over the surface of a structure following the introduction of temperature gradient through either active or passive methods. The presence of flaws will be indicated by the disruption of the normal pattern of heat flow as compared to the sound material. The transient temperature change will be captured by an infrared camera through one of the two different observation methods, namely transmission and reflection which offer different resolutions and results for defects of different depths and sizes. In order to enhance the sensitivity and detect ability, reference-free wave front technique [10] is explored to process the infrared images captured in order to detect possible thermal signature for kissing bonds of different depths.

\section{METHODOLOGY}

\section{Kissing-bond Specimens}

Two composite panels, one carbon-fiber composite laminate and the other glass-fiber laminate, were fabricated with matrix-defects to mimic kissing-bond at different depths below top surface as shown in

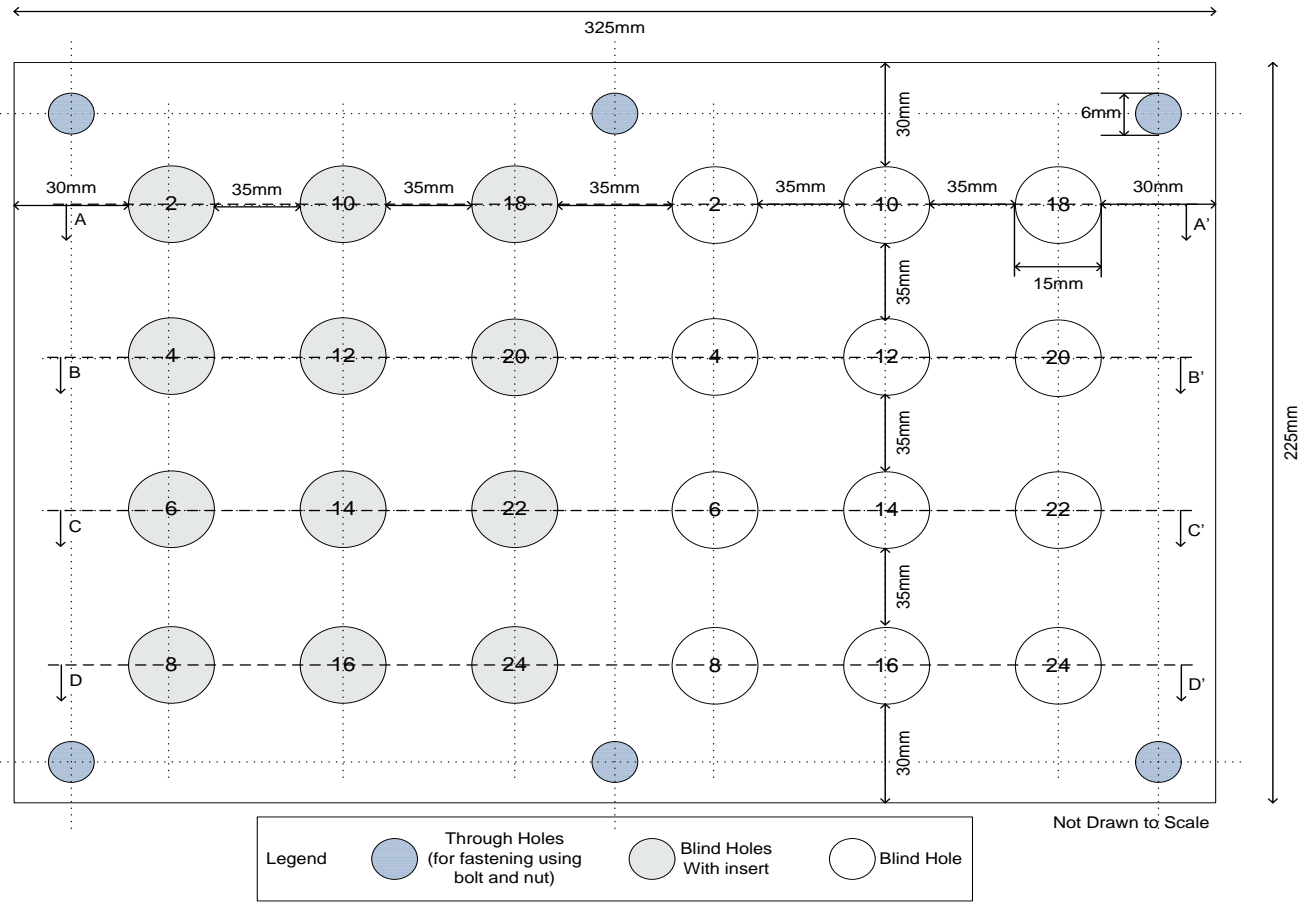


Fig.1, and were used in the study. The laminate is made up of two 26-ply panels fastened together by 6 bolts. Each panel measures $325 \mathrm{~mm}$ by $225 \mathrm{~mm}$. The top panel contains 24 defects which are arranged in 6 columns and 4 rows. As shown in Fig. 1a), counting from the left, the columns 4 to 6 contain blind holes which are drilled from the back of the panel to various depths, and columns 1 to 3 are blind holes but filled with inserts, as shown in Fig.2, of the same composite material with depths corresponding to the respective blind hole in columns 4 to 6 , to simulate kissing bonds. All the holes are $15 \mathrm{~mm}$ in diameter, and are numbered according to their depth, as shown in Fig.1b).
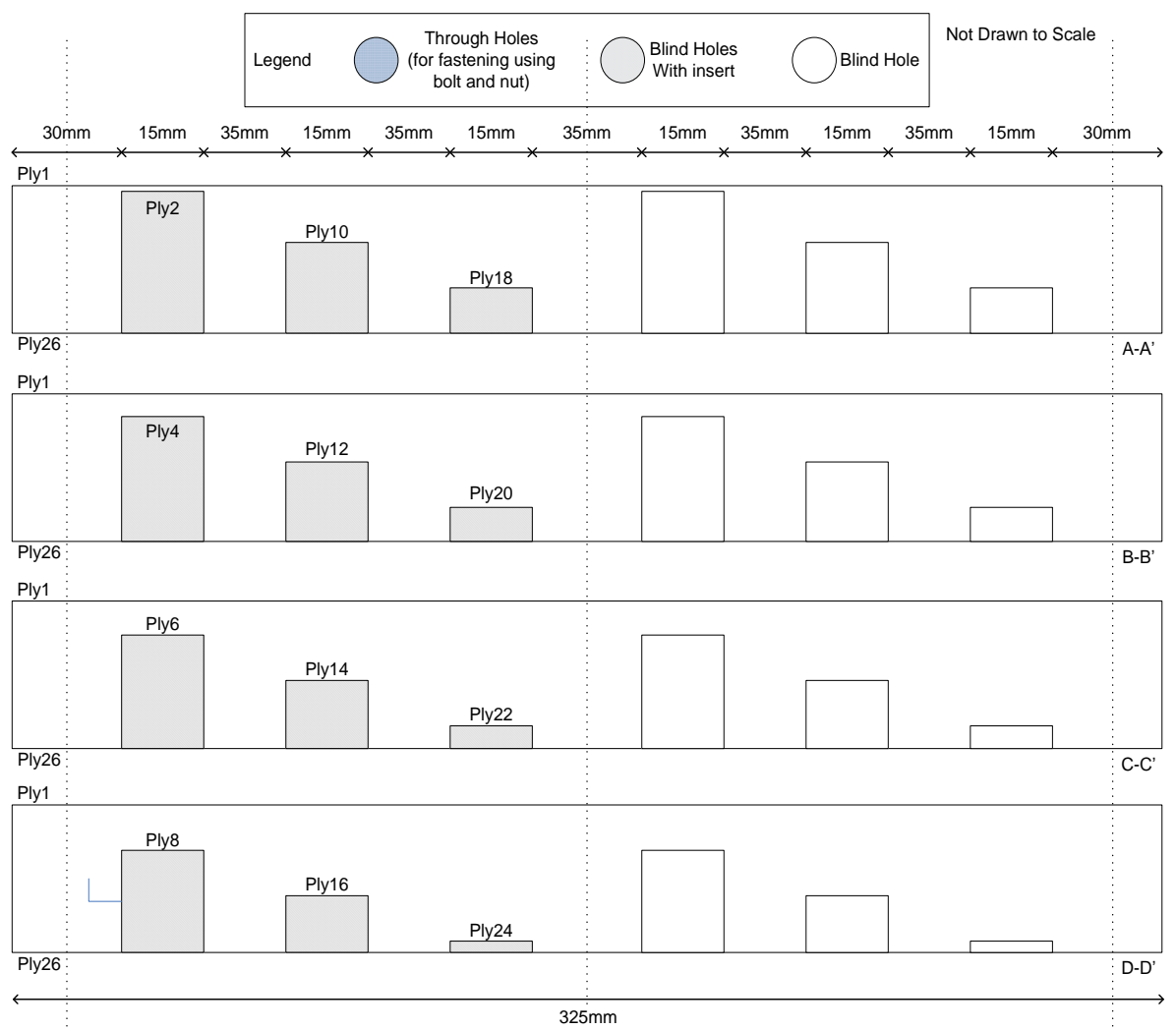

1b)

Fig. 1: Embedded matrix-defects in the composite specimens

The inserts can be then removed to apply proper adhesive layer in between the inserts and the laminate to simulate kissing bonds with adhesive layer (Fig. 2).

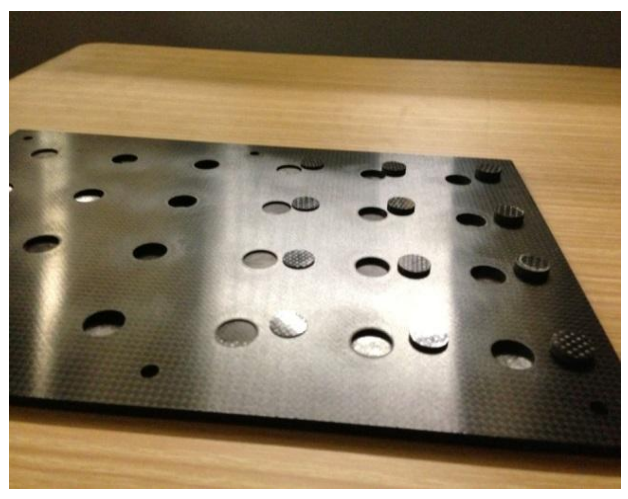

Figure 2: Inserts removed to apply adhesives 


\section{System configuration}

There are two general types of system configurations: transmission and reflection. In the transmission mode the camera and the lamp are in different side of the composite specimen as shown in Fig.3a), and in the reflection mode they are in the same side of the specimen as shown in Fig.3b). The specimen was hung up using 4 steel wires. In this way, during study, there is minimum direct physical contact with the specimen, and minimum heat transfer happen other than from heating lamp. The lamp and camera were placed at various distances from the specimen also at different angles in the different experiments for getting proper field of view and avoiding most direct reflection of the lamp in reflection mode.

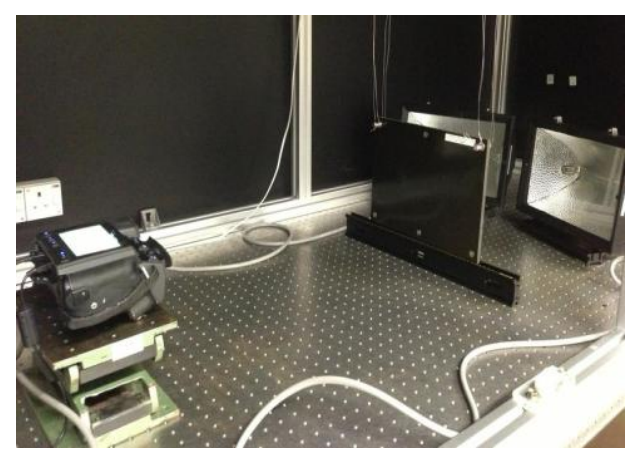

3a)

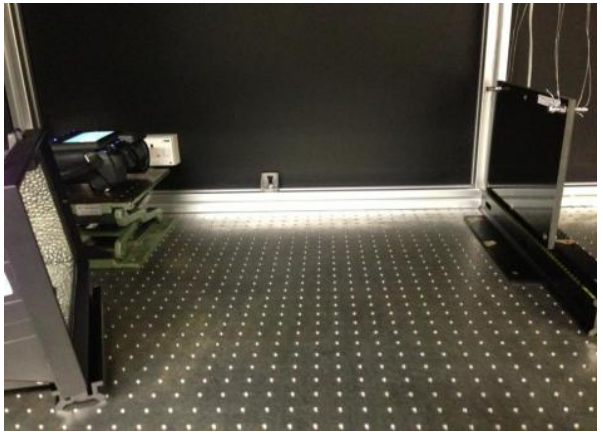

$3 b)$

Fig. 3 Lab set-up a) transmission mode and b) reflection mode

\section{Experimental Procedures}

The infrared thermal images in the heating-up and cooling-down process were recorded using an uncooled long wavelength infrared camera. The experimental procedures are as follows:

1. To position the infrared camera properly with the top surface facing to the camera and focusing to the area of interest to be examined;

2. To heat the specimen with the $1500 \mathrm{~W}$ halogen lamp, and recording images/video;

3. When surface temperature is up to about $55^{\circ} \mathrm{C}$, to switch off the lamp and block the lamp with a thick black cloth to avoid the residual radiation.

4. To stop the recording is stopped when the specimen is cooled sufficiently.

5. To export and process the raw data with our own thermal wave front processing algorithms and software.

\section{Signal Processing Algorithms and Software}

The camera used in the study is a long-wave un-cooled infrared camera that covers the wavelengths from 7 to $12 \mu \mathrm{m}$, and the camera software used is the FLIR ResearchIR software. It is capable of controlling the infrared camera directly. To eliminate the influence caused by the surface texture and emissivity to the surface temperature information, window-based sampling and differentiating algorithm is designed and implemented for infrared image processing and analysis where an adaptive digital windowing method is used to replace the physical lens-let array used in Shack-Hartmann wave front sensors [11, 12]. For thermal front processing, reference-free algorithm is used for detecting the first order derivatives of thermal front in $\mathrm{x}$ and $\mathrm{y}$ directions [10]. The whole processing software is coded using MatLab. 


\section{Results and Discussion}

\section{Mode of infrared image acquisition}

Both the transmission mode and reflection mode are investigated to examine the detectability of each mode of image acquisition and made a comparison thereafter. From the results, it can be seen that for the reflection mode, camera reflection is quite obvious and becomes a noise source. Direct reflection of heating lamp's broadband light emmision by laminate panel is another noise source, also mixes with the true signal from the composite specimen itself. The mixed infrared signal received by the camera makes the detectibility lowered in the heating up process. Also we observed that heating process produced significantly better results compared to the cooling process.

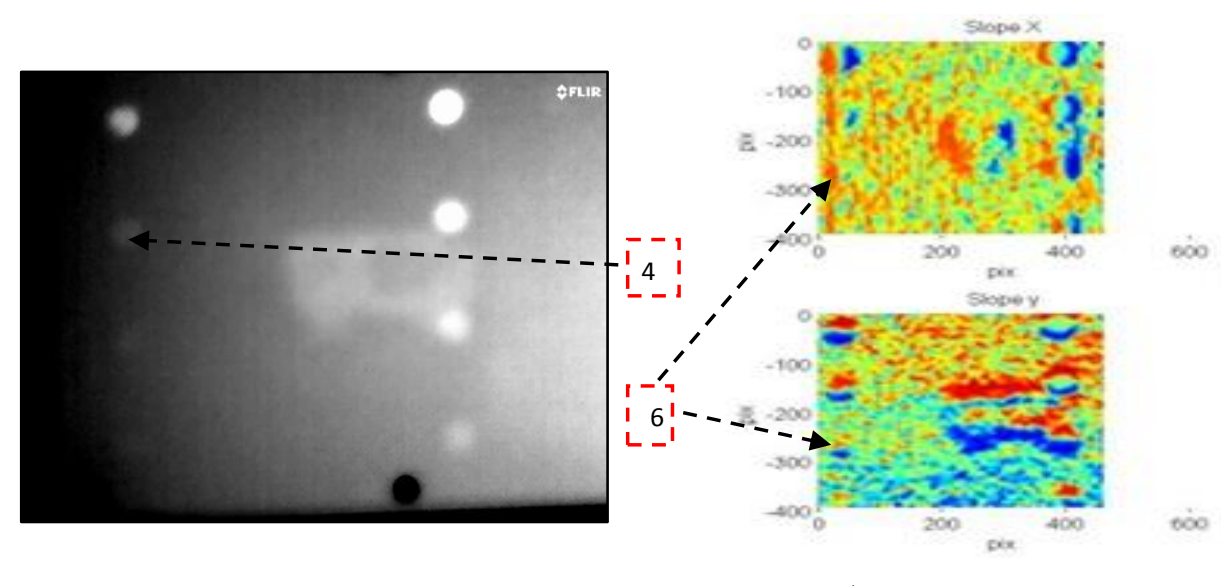

a) Raw IR image

b) $1^{\text {st }}$ derivative in $x$ and $y$

Fig. 4: A reflection mode inspection result of Carbon-fiber kissing bond specimen (left: raw temperature image; right: processed results with $1^{\text {st }}$ order derivative in $\mathrm{x}$ and $\mathrm{y}$

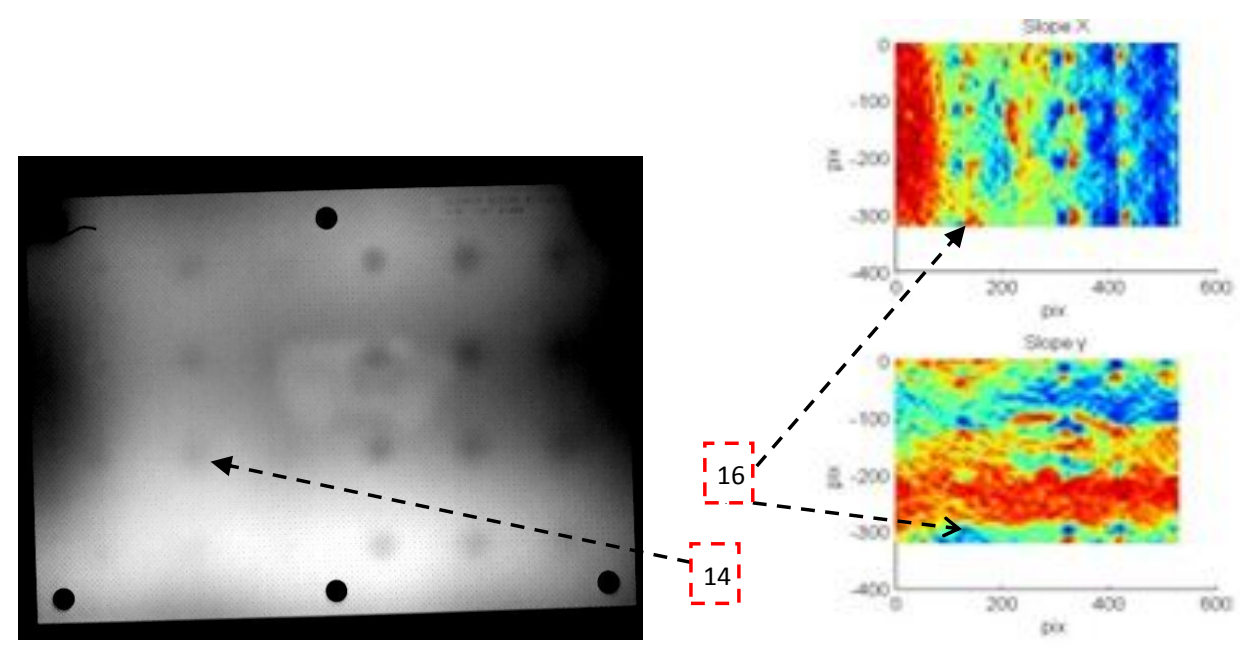

a) Raw IR image

b) $1^{\text {st }}$ derivative in $x$ and $y$

Fig. 5: A transmission mode inspection result of carbon-fiber kissing bond specimen using one halogen lamp

As shown in the raw IR images of Fig. 4 and 5, in the reflection result the kissing bond is detectable for up to number 4 , but in transmission mode number 12 kissing bond is detectable. If using the $1^{\text {st }}$ derivative result to evaluate, kissing bond up to numbered 6 can be detected in reflection mode, whereas in transmission mode, up to numbered 16 is detectable. The detectibility is improved by calculating $1^{\text {st }}$ order derivative. 


\section{Rate of heating}

Heating the specimen with two halogen lamps increased the heating rate of the specimen. The time taken for the temperature of the specified point on the specimen to change from $30^{\circ} \mathrm{C}$ to $55^{\circ} \mathrm{C}$ was 156 seconds when two lamps were used and 243 seconds when only one lamp was used. The result with one lamp is shown in Fig.5, and that with two lamps is shown in Fig.6.

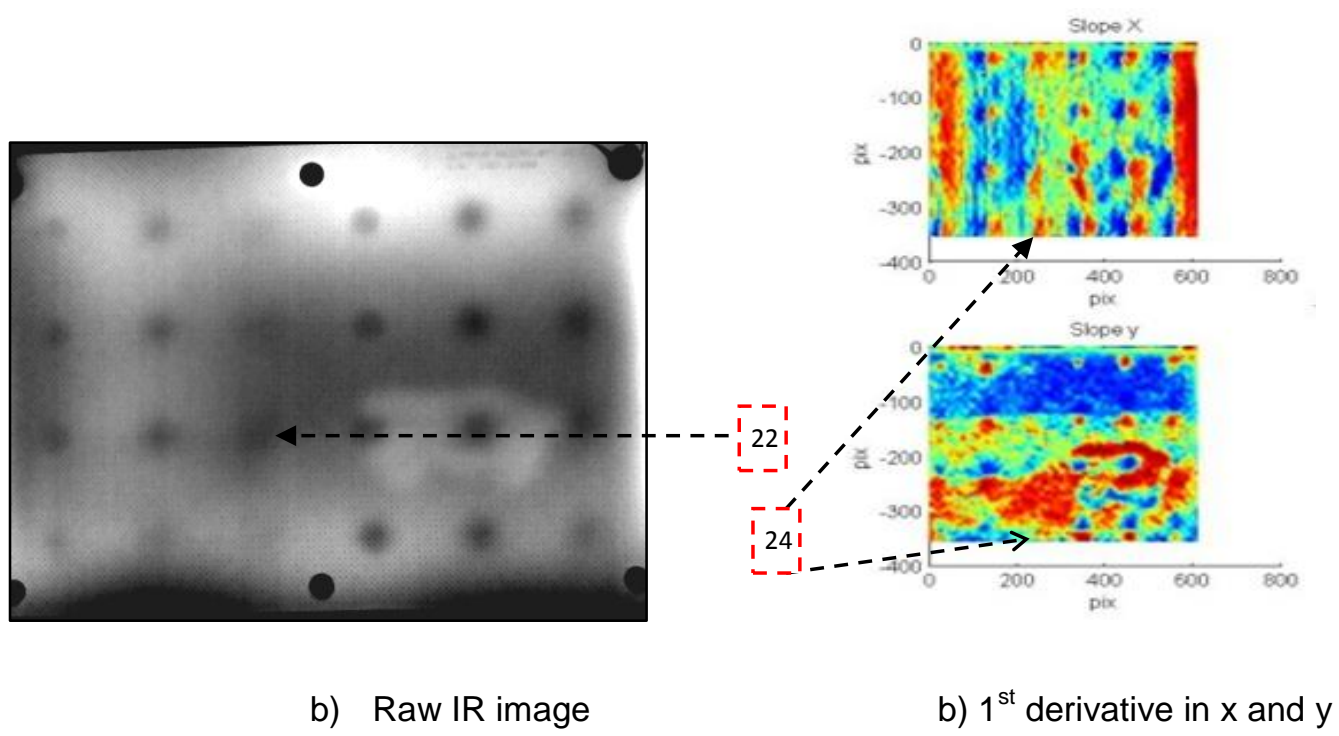

Fig. 6: A transmission mode inspection inspection result of carbon-fiber kissing bond specimen using two halogen lamps

The increased heating rate increased the contrast between the defective and sound areas. This was probably because the thermal transfer in the direction parallel to the plane was slower and thus the area adjacent to the trapped air in the defects did not have sufficient time to heat up, showing a notable contrast compared to the surrounding area. Comparing Figs. 5 and 6, the raw image of Fig. 6 had a better indication of the defects than that in Fig.5, and all the kissing bond could be identified in the processed derivative in $x$ direction. Kissing bonds 18, 20, 22 and 24 which could not be identified in Fig. 5 can be observed from Fig. 6 , especially clear and obvious in $x$ derivative, all the defects can be identified easily.

For the glass-fiber specimens, similar study is done and the results showed that more powerful heating can enhance the visibility and detect ability of kissing bond defects.

\section{Presence of adhesive glue layer}

Glue was added in between the holes and inserts of the specimen to more closely simulate the properties of kissing bonds. Transmission and reflection experiments using one lamp was done on the specimen with glue added. As shown in Fig. 7, reflection mode results show that the defects were significantly less visible after the glue was added in the glass-fiber specimen, and such kissing bond defects could barely be seen. 


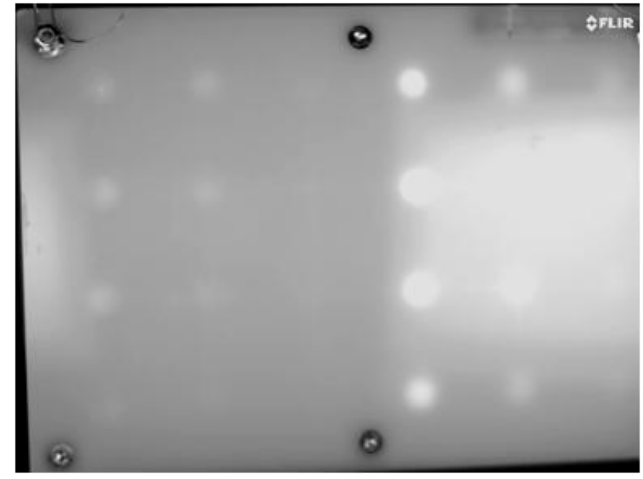

7a)

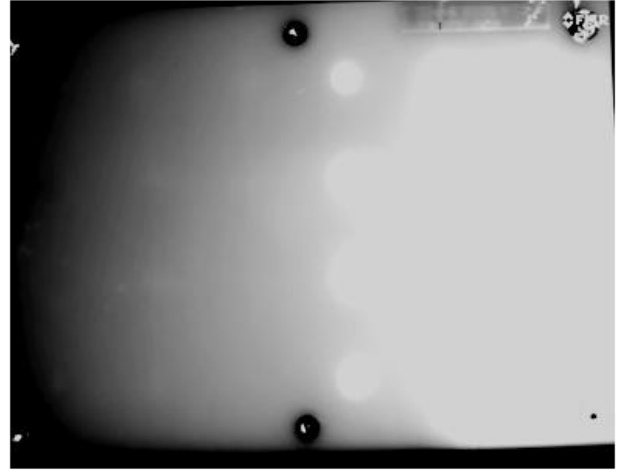

7b)

Fig.7 Raw image of reflection mode for glass-fiber specimen without glue in a) and with glue in b).

For the transmission mode, From the result obtained for carbon-fiber kissing-bond specimen with glue as shown in Fig. 8 and that without glue in Fig.5, we can see that the two results do not show significant difference in the detectability. Both are able to detect up to kissing bonds number 16 and number 22 is slightly seen in the derivative result. The similar situation occurred for the glass-fiber specimen. The original design of the kissing-bond specimen without glue between the inserts and substrate mimics the kissing bond with no bonding, and after putting normal glue in it simulates the kissing bond with weak/little bonding strength. Our IR NDT results show that our IR inspection system seems able to detect both of two kissing bond scenarios.

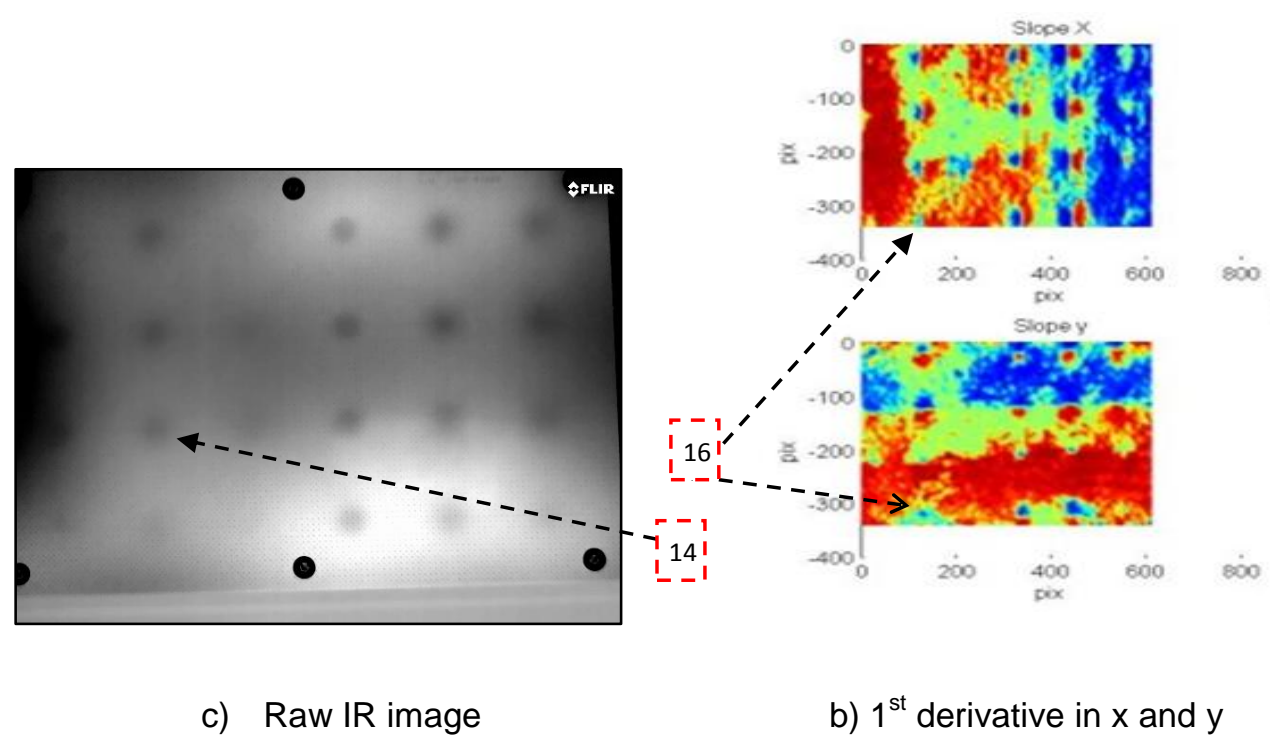

Fig. 8: A transmission mode inspection results of Carbon-fiber kissing bond specimen with adhesive glue

\section{Conclusions}

In this paper, the investigation of detectibility of the kissing bonds in the carbon-fiber and glass-fiber reinforced polymer panels is presented. By analysing the infrared images with reference free approach, $1^{\text {st }}$ derivatives in $\mathrm{x}$ and $\mathrm{y}$ directions are calculated. Our study showed that the detectability of the system was improved by increasing the rate of heating, and the transmission mode has higher detectibility than the reflection mode. With adhesive glue layer added to the specimen, the detectibility 
is slightly decreased. Overall, all the kissing bonds and blind holes could be detected by heating the specimen with two halogen lamps in transmission mode. Compare the analyzed derivative profile and raw IR image, it can be found that detectibility is improved up to 2 to 6 number, that means the defects at 2-6 more plies deeper can be detected with the derivative profile. Following effort and work will be put into further analysis the derivative profiles in two directions, and try to generate robust thermal signature of kissing-bond defects.

\section{References:}

[1] "Boeing: Program Fact Sheet," 2013. [Online]. Available: http://www.boeing.com/boeing/commercial/787family/programfacts.page. [Accessed 23 May 2013].

[2] Composite resources . (2012). Retrieved June 13, 2013, from http://www.compositeresources.com/composites/advantages/

[3] Ajay Kapadia, T. L. (n.d.). Non Destructive Testing of Composite Materials. National Composites Network .

[4] G. Guo, J. Tu, Y. Zhang and Y. Shi, "Inspection of Kissing-bond Defect in Honeycomb Structure By Shearography," in 18th World Conference on Nondestructive Testing, Durban, 2012.

[5] B. Ehrhart, B. V. (2010). Methods for the Quality Assessment of Adhesive Bonded CFRP Structures.

[6] K. A. Tsoi and N. Rajic, "Non-destructive Evaluation of Aircraft Structural Components and Composite Materials at DSTO Using Sonic Thermography," DSTO Defence Science and Technology Organisation, 2011.

[7] F.J. "The effect of compressive loading on the ultrasonic detectability of kissing bonds in adhesive joints". Journal of Nondestructive Evaluation, 95-104. (2002).

[8] S. Marinetti, Y. A. Plotnikov, W. P. Winfree and A. Braggiotti, "Pulse phase thermography for defect detection and visualization," 1999.

[9] B. Zadler, J. H. Le Rousseau, J. A. Scales and M. L. Smith, "Resonant ultrasound spectroscopy: Theory and Application".

[10] L. Zhao, W. G. (2011). Reference-free Shack-Hartmann wavefront sensor. Optics Letters

[11] L. Zhao, B. I. Pang and Z. Zhong, "Thermal differential sensing for defect inspection of carbon fiber reinforced polymer (CFRP) composite laminate," in 11th International Conference on Quantitative InfraRed Thermography, 2012.

[12] W. Guo, L. Zhao, S. T. Chin, I.-M. Chen and S. C. Joshi, "Adaptive centroid-finding algorithm for freeform surface measurements," Applied Optics, vol. 52, no. 10, pp. D75 - D83, 2013. 